\title{
A RAPID AND SENSITIVE LIQUID CHROMATOGRAPHY- MASS SPECTROMETRY/MASS SPECTROMETRY METHOD FOR ESTIMATION OF PIOGLITAZONE, KETO PIOGLITAZONE AND HYDROXY PIOGLITAZONE IN HUMAN PLASMA
}

\author{
REVATHI NAGA LAKSHMI PONNURI ${ }^{*}$, PRAHLAD PRAGALLAPATI ${ }^{2}$, RAVINDRA N ${ }^{3}$, \\ VENKATA BASAVESWARA RAO MANDAVA ${ }^{4}$
}

\begin{abstract}
${ }^{1}$ Department of Pharmacy, Faculty Pharmaceutical Sciences, Pharmaceutical Analysis, Krishna University, Machilipatnam - 521002, Andhra Pradesh, India. ${ }^{2}$ Department of Pharmaceutics, Acharya Nagarjuna University, Guntur - 522 510, Andhra Pradesh, India. ${ }^{3}$ Department of Pharmaceutical Chemistry, Chilkur Balaji College of Pharmacy, Hyderabad - 500 075, Telangana, India. ${ }^{4}$ Department of Chemistry, Faculty of Sciences, Directorate of Admissions, Krishna University, Machilipatnam - 521 002, Andhra Pradesh, India. Email: pharma_revathi@yahoo.com
\end{abstract}

Received: 27 May 2017, Revised and Accepted: 17 August 2017

ABSTRACT

Objective: The main objective of the work was to develop a straightforward, fast and selective liquid chromatography/tandem mass spectrometry (LC-MS/MS) assay for determination of pioglitazone (PG), keto pioglitazone (KPG), and hydroxy pioglitazone (HPG) in human plasma and to validate as per recent guidelines.

Methods: Analyte and the internal standard (IS) were extracted from plasma through liquid-liquid extraction and chromatographed on a Xterra RP18, $100 \times 4.6,5 \mu$ column using methanol: acetonitrile mixture and $10 \mathrm{mM}$ Ammonium formate buffer (70:30, v/v) as the mobile phase at a flow rate of $0.7 \mathrm{~mL} / \mathrm{min}$. The API-3200 Q Trap LC-MS/MS instrument in multiple reaction monitoring mode was used for detection. Diphenhydramine was utilized as IS.

Results: The linearity was established in the concentration range of $20.15-1007.58 \mathrm{ng} / \mathrm{mL}$ for PG, 20.35-1017.58 ng/mL for KPG, and 19.68-491.22 ng/mL for HPG in human plasma. All the validation parameters were well within the acceptance limits.

Conclusion: A new simple LC-MS/MS method was developed for the determination of PG, KPG, and HPG in human plasma. This method can be easily applied for the estimation of pharmacokinetic parameters of PG, KPG, and HPG.

Keywords: Pioglitazone, Keto pioglitazone, Hydroxy pioglitazone, Human plasma, Method validation, Liquid chromatography/tandem mass spectrometry.

(C) 2017 The Authors. Published by Innovare Academic Sciences Pvt Ltd. This is an open access article under the CC BY license (http://creativecommons. org/licenses/by/4. 0/) DOI: http://dx.doi.org/10.22159/ajpcr.2017.v10i12.20284

\section{INTRODUCTION}

Pioglitazone (PG) is used for the treatment of diabetes mellitus type 2 . Chemical name of PG is ( \pm )-5-[p-[2-(5-ethyl-2-pyridyl)ethoxy]benzyl]2,4-thiazolidinedione monohydrochloride (Fig. 1) and chemical formula is $\mathrm{C}_{19} \mathrm{H}_{20} \mathrm{~N}_{2} \mathrm{O}_{3}$ S.HCl. PG is a thiazolidinedione antidiabetic agent that depends on the presence of insulin for its mechanism of action. PG decreases insulin resistance in the periphery and the liver resulting in increased insulin-dependent glucose disposal and decreased hepatic glucose output. Unlike sulfonylureas, PG is not an insulin secretagogue. PG is a potent and highly selective agonist for peroxisome proliferators - activated receptor - gamma (PPAR $\gamma$ ). PPAR receptors are found in tissues important for insulin action such as adipose tissue, skeletal muscle, and liver. Activation of PPAR $\gamma$ nuclear receptors modulates the transcription of a number of insulin responsive genes involved in the control of glucose and lipid metabolism [1]. Keto pioglitazone (KPG), a metabolite of PG with the molecular formula: $\mathrm{C}_{19} \mathrm{H}_{18} \mathrm{~N}_{2} \mathrm{O}_{4} \mathrm{~S}$ and hydroxy pioglitazone (HPG) is another PG metabolite with the molecular formula: $\mathrm{C}_{19} \mathrm{H}_{20} \mathrm{~N}_{2} \mathrm{O}_{4} \mathrm{~S}$.

As per the literature, various analytical methods such as membrane sensitive electrode [2], potentiometric [3], ultravioletspectrophotometric [4], high-performance liquid chromatographic (HPLC) methods [5-10], ultra-performance liquid chromatograph method [11], and liquid chromatography-mass spectrometry (LCMS) methods [12-17] have been reported for the determination of PG and its metabolites. The particular described methods have to sacrifice time, resolution as well as sensitivity. Today, there exists a prerequisite to develop fast or ultra-fast methods such as LC-MS/MS without any loss of separation efficiency and sensitivity. To date, just one LC-MS/MS method happen to be noted for the determination of PG and its active metabolites KPG and HPG in human plasma samples.

In this paper, the author's details an effective LC-MS/MS assay method for the determination of PG, KPG, and HPG in human plasma using diphenhydramine as an internal standard (IS) to avoid the possible matrix effect related problems and variability in recovery between analytes and the IS. There should be a suitable analytical assay with greater sensitivity to estimate the concentrations at elimination phase of the pharmacokinetic profile. The validated method can be successfully applied to a pharmacokinetic study in humans, and obtained results were authenticated through incurred samples reanalysis. Authors of this paper have also published other simultaneous analytical methods $[18,19]$.

\section{METHODS}

\section{Chemicals and reagents}

The reference samples PG, KPG, and HPG were obtained from Dr. Reddy's Laboratories Ltd. (Hyderabad, India). Acetonitrile and methanol were of HPLC grade purchased from Rankem. Similarly, analytical grade ammonium acetate was from Merck Ltd (Mumbai, India). Ultra-pure water was prepared using Milli $Q$ water purification (Millipore, Bangalore, India). 


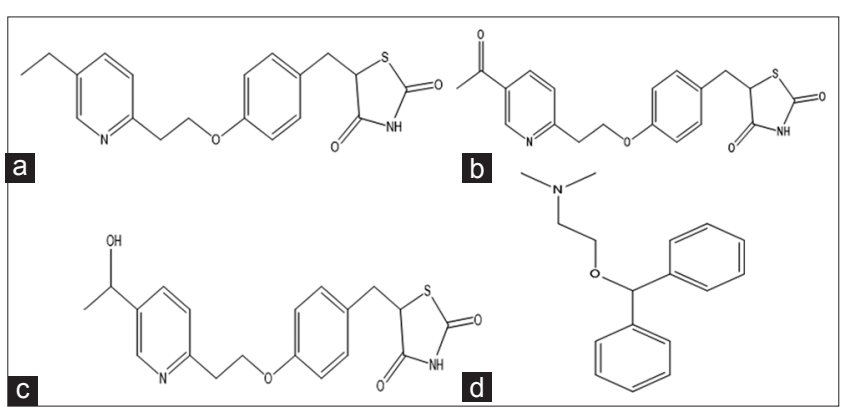

Fig. 1: Chemical structures of (a) pioglitazone, (b) keto pioglitazone, (c) hydroxy pioglitazone, and (d) diphenhydramine

\section{LC-MS/MS instrument and conditions}

An HPLC system (Shimadzu, Kyoto, Japan) equipped with a Xterra RP18, $100 \times 4.6 \mathrm{~mm}, 5 \mu \mathrm{m}$ column, a binary LC-20AD prominence pump, an auto sampler (SIL-HTc) and a solvent degasser (DGU-20A ( $_{3}$ was used for the study. Aliquot of $20 \mu \mathrm{L}$ of the extracted samples were injected into the column. Column oven temperature was maintained at ambient temperature $\left(40 \pm 2^{\circ} \mathrm{C}\right)$. Methanol: Acetonitrile $(1: 1 \mathrm{v} / \mathrm{v})$ mixture and $10 \mathrm{mM}$ Ammonium formate buffer (70:30, v/v) were used as the mobile phase at a flow rate of $0.7 \mathrm{~mL} / \mathrm{min}$. API 3200 (Q-Trap) mass spectrometer (Applied Biosystems) equipped with a Turboionspray ${ }^{\mathrm{TM}}$ in positive ion mode was used for the quantification of the analyte.

\section{Preparation of spiked plasma samples}

Stock solution of PG, KPG, and HPG was prepared in HPLC grade methanol $(1 \mathrm{mg} / \mathrm{mL})$. Two separate stock solutions were used for the preparation of calibration curve (CC) standards and quality control (QC) samples. A mixture of methanol and water (50:50, v/v; diluent) was used to prepare naftopidil working solutions. The working concentration of the diphenhydramine $(1000 \mathrm{ng} / \mathrm{mL})$ was prepared using the same diluent.

Calibration samples and QC samples were prepared in $\mathrm{K}_{2}$ EDTA human plasma as a bulk and stored at $-70 \pm 10^{\circ} \mathrm{C}$. Calibrates were prepared at eight concentration levels of $\mathrm{PG}$ and $\mathrm{KPG}$ ranging from 20.0 to $1000 \mathrm{ng} / \mathrm{mL}$ and $\mathrm{HPG}$ ranging from 20.0 to $500 \mathrm{ng} / \mathrm{mL}$ with interference free $\mathrm{K}_{2}$ EDTA plasma using final concentration as a single batch at each concentration. Likewise, QC samples at concentrations of PG and KPG ranging from 20.0 to $850 \mathrm{ng} / \mathrm{mL}$ and $\mathrm{HPG}$ ranging from 20.0 to $850 \mathrm{ng} / \mathrm{mL}$.

\section{Extraction protocol}

With draw plasma blank, CC standards, QC samples from deep freezer, and allow them to thaw at room temperature. Vortex the thawed samples to ensure complete mixing of contents.

$$
\begin{gathered}
\text { Extraction procedure: LLE } \\
\downarrow
\end{gathered}
$$

Aliquot $0.20 \mathrm{~mL}$ of plasma sample into a pre labeled RIA vial, add 50 $\mu \mathrm{L}$ of ISTD $(1 \mu \mathrm{g} / \mathrm{mL})$ working stock solution and, vortex. $\downarrow$

To the above sample add $2 \mathrm{~mL}$ of ethyl acetate $\downarrow$

Vortex the sample for $5 \mathrm{~min}$ at $2500 \mathrm{rpm}$ for complete mixing

Centrifuge for $5 \mathrm{~min}$ at $4500 \mathrm{rpm}$ at $5^{\circ} \mathrm{C}$

$$
\downarrow
$$

Remove the supernatant into pre labeled RIA vial and evaporate under

$$
\downarrow
$$

nitrogen gas at $40^{\circ} \mathrm{C}$ to dryness

$$
\downarrow
$$

Reconstitute with $1.0 \mathrm{~mL}$ of mobile phase and vortex the samples for 30 seconds

\section{Load the samples into auto injector according to the batch $\downarrow$ \\ Inject $20 \mu \mathrm{L}$ onto analytical column}

\section{Method validation parameters}

A thorough and complete method validation of PG, KPG, and HPG in human plasma was carried out as per US FDA [20] and EMEA [21] guidelines. The actual parameters determined were carryover test, selectivity, matrix effect, sensitivity, linearity, precision and accuracy, recovery, dilution integrity, run size evaluation, ruggedness, and stability.

The selectivity of the method was assessed in six different sources of plasma, of which, six were normal $\mathrm{K}_{2}$ EDTA plasma. Carryover test ended up being conducted to substantiate any carryover associated with analyte as well as the IS actually, which may reveal throughout following goes. The carryover test samples were injected in the following sequence, i.e., MP®AQS ULOQ®MP®Extracted blank®Extracted ULOQ®Extracted blank®MP. Sensitivity was determined by analyzing six replicates of plasma samples spiked with the lowest level of the CC concentrations (lower limit of quantification [LLOQ]). Matrix effect was assessed by comparing the mean area response of post-extraction spiked samples with a mean area of aqueous samples (neat samples) prepared in mobile phase solutions at lower quality control (LQC) and higher quality control (HQC) levels. The overall precision of the matrix factor was expressed as coefficient of variation (CV).

Matrix $=\frac{\text { Peak response area ratio in presence of matrix ions }}{\text { Mean peak response area ratio in absence of matrix ions }}$

The linearity of the proposed method was determined by analysis of standard CCs containing eight non-zero concentrations along with one blank plasma sample and one zero standard (blank sample with the IS). Each CC was analyzed individually by least square weighted $\left(1 / x^{2}\right)$ linear regression. Intraday precision and accuracy results were determined using six replicates of LLOQ QC, LQC, middle quality control (MQC), and HQC samples analyzed on the same day. Interday precision and accuracy were measured by analyzing five different analytical batches on 3 consecutive days. Extraction recoveries of analyte were determined at LLOQ QC, LQC, MQC, and HQC by comparing the peak area of extracted analyte sample with the peak area of non-extracted standard. Similarly, recovery of IS was determined at a concentration of $1000.00 \mathrm{ng} / \mathrm{mL}$. The diluted samples were processed and analyzed using un-diluted CC standards. The ruggedness of the method verified by analyzing one precision and accuracy batch on another instrument of the same make using different column (different batch no.) and processed by another analyst.

The stock solution stability at room temperature and at $2-8^{\circ} \mathrm{C}$ in the refrigerator was performed by comparing the area response stability samples with the response of the sample prepared from fresh stock solution. The solutions were considered stable if the deviation within $\pm 10 \%$ from nominal value. Bench top stability at room temperature (6.3 hrs), processed samples stability (auto sampler stability for $35 \mathrm{hrs,}$ dry extract stability for $24 \mathrm{hrs}$, and reinjection stability for $52 \mathrm{hrs}$ ), freeze-thaw stability were performed at LQC and HQC levels using six replicates at each level. The stability samples were processed and quantified against freshly spiked CC standards along with freshly spiked QC samples. Samples were considered to be stable if assay values were within the acceptable limits of accuracy ( $\pm 15 \%$ standard deviation) and precision ( $£ 15 \%$ relative standard deviation).

\section{RESULTS AND DISCUSSION}

\section{Mass spectrometry}

To investigate the pharmacokinetics, tolerability, and safety of newer formulations, one should have an appropriate analytical method for its identification and quantification. Nowadays rapid and highly sensitive 
analytical technique such as LC-MS is most widely used in bio-analysis of drugs and metabolites. The proposed LC-MS/MS method is developed with the aim to quantify the PG, KPG, and HPG concentrations. During the method, development stages mass spectrometric and chromatographic conditions critically evaluated to obtain a good and reproducible response with a better resolution from the end ogenous components. During tuning of analytes high intense signals were obtained in positive ion mode than the negative mode for the analyte and the IS due to their ability to accept the protons. Furthermore, the adequate and reproducible response was obtained by changing the source dependent parameters. Dwell time was set at $200 \mathrm{~ms}$, at which no cross talk was found. The positive ion spray mass spectrum revealed a protonated molecular by monitoring the transition pairs of $\mathrm{m} / \mathrm{z} 357.20$ precursor ion to the $\mathrm{m} / \mathrm{z} 134.20$ product ion for PG; $\mathrm{m} / \mathrm{z} 371.00$ precursor ion to the $\mathrm{m} / \mathrm{z} 148.20$ product ion for $\mathrm{KPG} ; \mathrm{m} / \mathrm{z} 373.00$ precursor ion to the $\mathrm{m} / \mathrm{z} 150.20$ product ion for HPG, and $\mathrm{m} / \mathrm{z} 256.30$ precursor ion to the $\mathrm{m} / \mathrm{z} 167.30$ product ion for the IS. Data acquisition was performed with Analyst Software ${ }^{\mathrm{TM}}$ (version 1.6.1) in the multiple reaction monitoring (MRM) mode (Table 1).

\section{Chromatographic conditions}

Acetonitrile and methanol are widely used an organic modifier for LC-MS analysis. Hence, during method development, both the solvents were checked in combination with acidic buffers such as ammonium acetate, ammonium formate, formic acid, and acetic acid. The response obtained with methanol and $10 \mathrm{mM}$ ammonium formate as a mobile phase was good; but not reproducible. Moreover, a variety of chromatographic columns such as $\mathrm{C}_{8}$ and $\mathrm{C}_{18}$ of different makes (Kromasil 100-5 C18, 50×4.6 mm, $5 \mu \mathrm{m}$; Discovery HS $\mathrm{C}_{18} 50 \times 4.6 \mathrm{~mm}$, $5 \mu \mathrm{m}$; Alltima HP C $180 \times 4.6,3 \mu \mathrm{m}$; Kromasil 100-5C ${ }_{18}, 100 \times 4.6,5 \mu \mathrm{m}$; Zorbax SB C ${ }_{18}, 50 \times 4.6,5 \mu \mathrm{m}$; Zorbax XDB-phenyl $75 \times 4.6,3.5 \mu \mathrm{m}$; Ace $3 \mathrm{C}_{18} 150 \times 4.6,3 \mu \mathrm{m}$; Hypurity advance $75 \times 4.6,5 \mu \mathrm{m}$ ) were verified to achieve adequate retention time with short run time, better separation from endogenous components, symmetric peak shape and satisfactory response for the analyte. The best chromatographic conditions were achieved with methanol: Acetonitrile (1:1) and $10 \mathrm{mM}$ ammonium formate $(70: 30, v / v)$ as a mobile phase under isocratic conditions. Xterra RP18 100×4.6 mm column gave good peak shape (Figs. 2-4). The mobile phase flow rate was set at $0.7 \mathrm{~mL} / \mathrm{min}$, which can produce better acceptable chromatographic peak shape and short run time of $4.0 \mathrm{~min}$ (Table 2).

\section{Extraction procedure optimization}

The reported procedures have employed solid phase extraction technique for the sample preparation. A sensitive analytical method

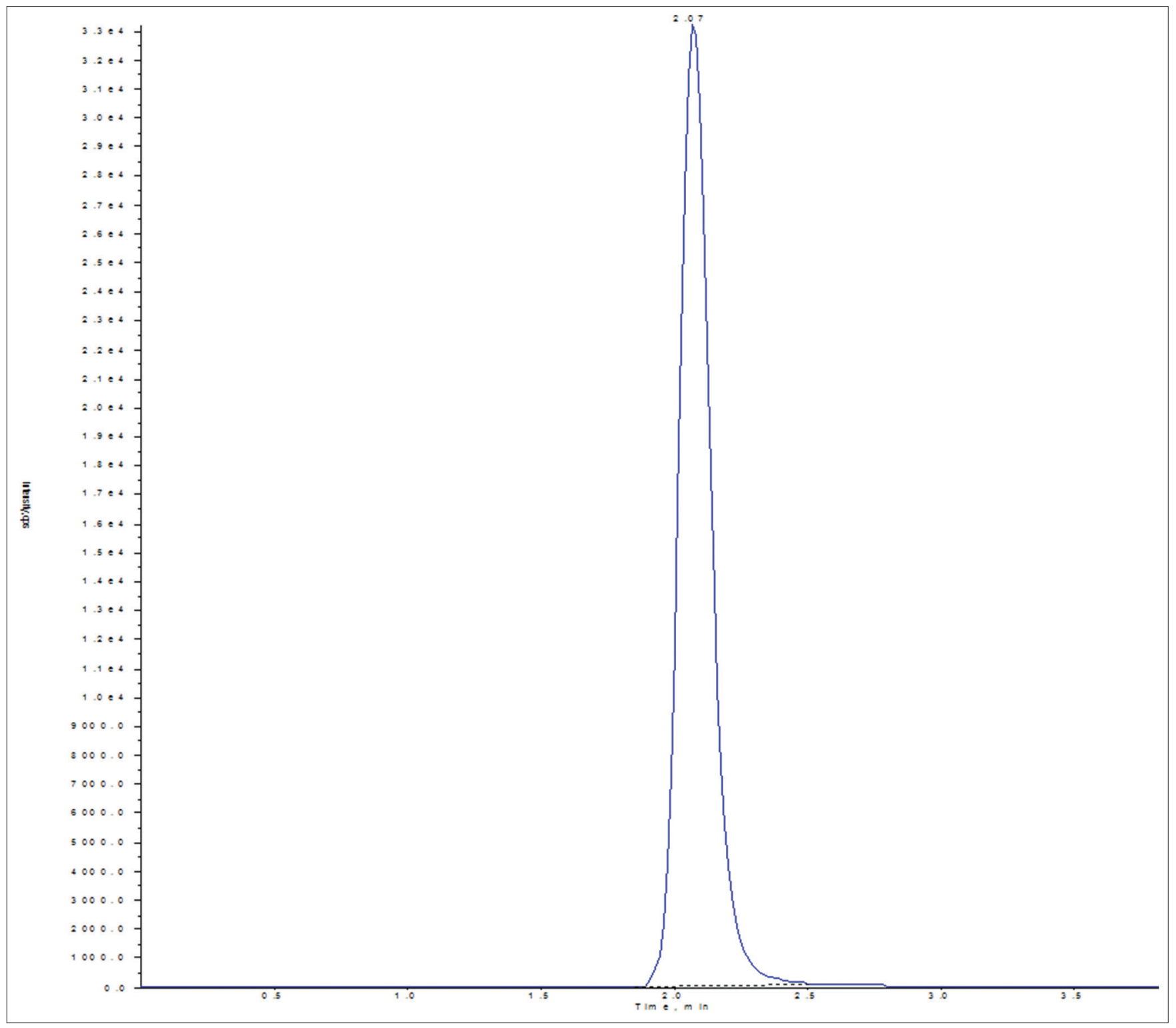

Fig. 2: Representative chromatogram of zero standard sample of pioglitazone 


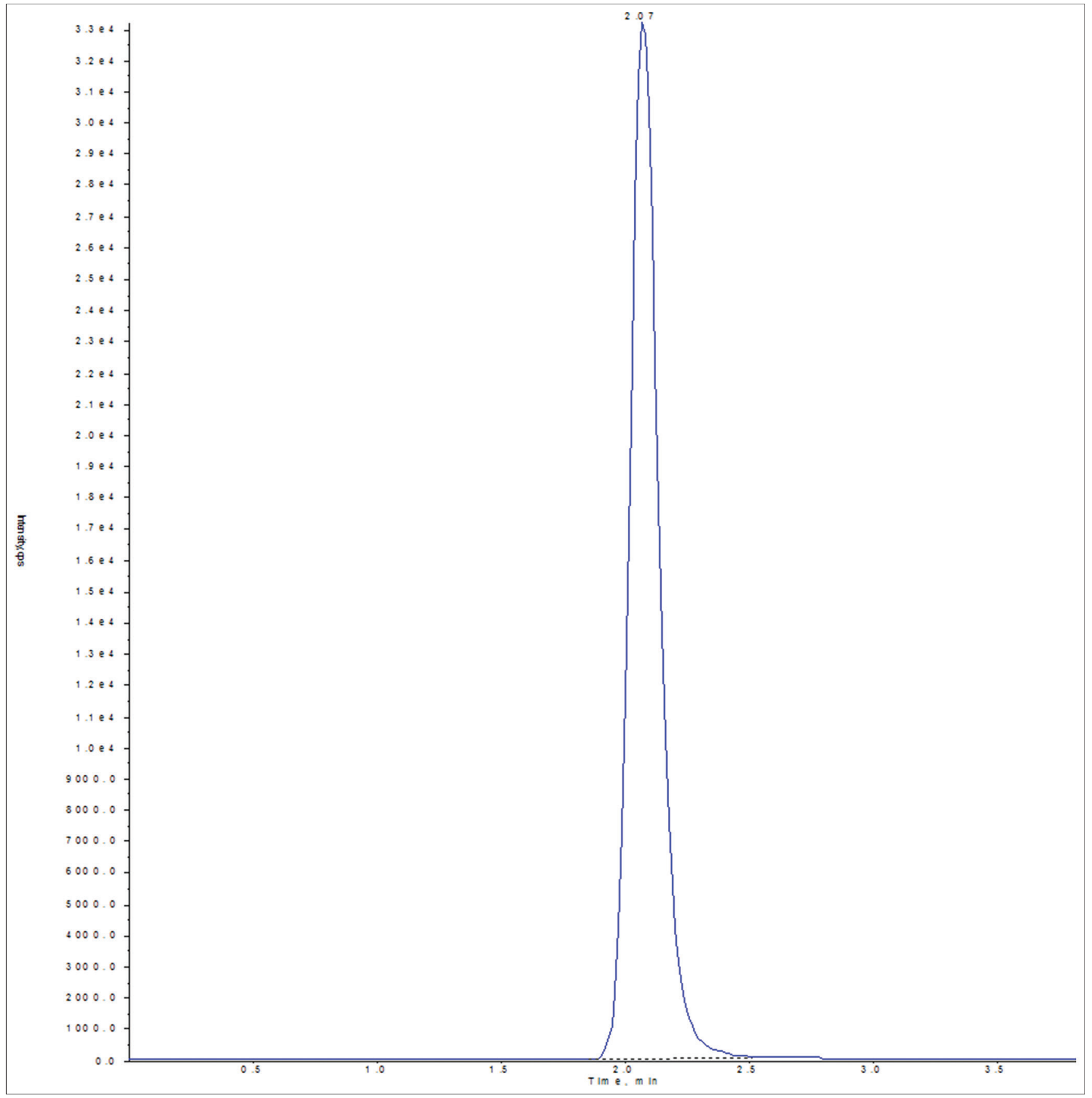

Fig. 3: Representative chromatogram of zero standard sample of keto pioglitazone

in biological samples requires a judicious selection of extraction procedure which can harvest high recovery with negligible or no matrix effect. Therefore, locally linear embedding (LLE) was tried.

\section{Weighting factor of regression method}

To determine whether to fit the data for the CCs by weighted or unweighted linear regression, the functional dependence of the standard deviation of the PG/diphenhydramine, KPG/diphenhydramine, and HPG/ diphenhydramine and area ratio on sample concentration is evaluated. It was found that best fit and weighting is linear with offset $1 / \mathrm{X}^{2}$.

\section{Autosampler carry over}

Carryover test was performed in the following sequence. MP®AQS ULOQ $® M P \circledast$ Extracted blank $®$ Extracted ULOQ $®$ Extracted blank $® M P$. No significant injector carryover is observed.

\section{Selectivity}

Selected blank human $\mathrm{K}_{2}$ EDTA plasma sources was carried through the extraction procedure and chromatographed to determine the extent to which endogenous human $\mathrm{K}_{2}$ EDTA plasma components may contribute to chromatographic interference with the PG, KPG, and HPG, and the IS. No significant interference is observed in six different lots of human $\mathrm{K}_{2}$ EDTA plasma samples.

\section{Sensitivity}

Six LLOQ samples were prepared independent of CC standards. All the results were found within the limits.

CCs

CCs were found to be consistently accurate and precise over the range of 20.15-1007.58 ng/mL for PG, 20.35-1017.58 for KPG, and 19.68-491.22 


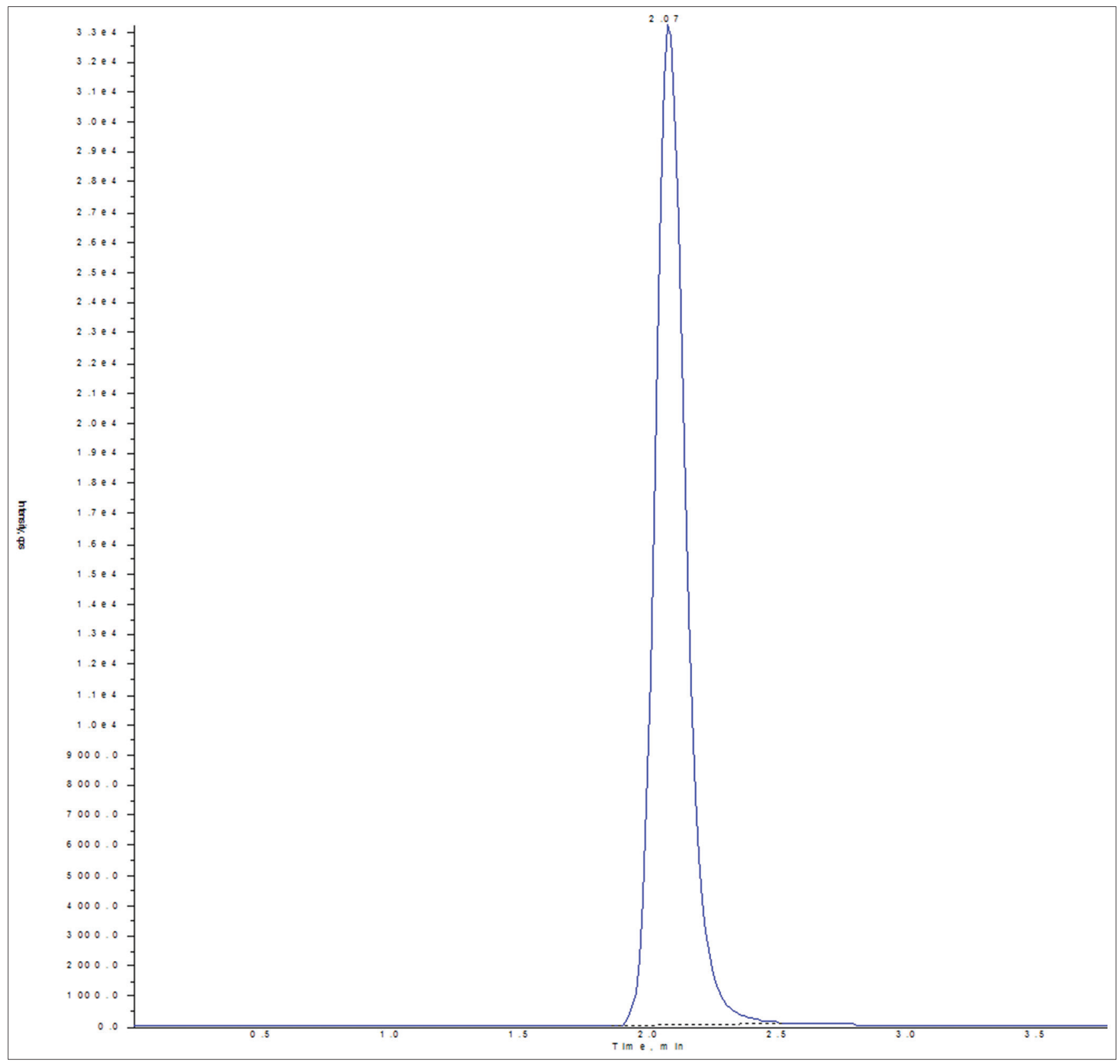

Fig. 4: Representative chromatogram of zero standard sample of hydroxy pioglitazone

for HPG. The regression coefficient ( $\mathrm{r}$ ) is $\geq 0.9800$. Back calculations were made from the CCs to determine PG, KPG, and HPG concentrations of each calibration standard.

\section{Interday accuracy and precision}

The interday accuracy and precision evaluation were assessed by the repeated analysis of human $\mathrm{K}_{2}$ EDTA plasma samples containing different concentrations of PG, KPG, and HPG on separate occasions. A single run consisted of a CC, six replicates of LLOQ LQC, MQC, and HQC samples.

The interday coefficients of variation ranged from 2.64 to $6.15,5.34$ to 6.05, and 3.15 to 7.17 for $P G, K P G$, and $\mathrm{HPG}$, respectively. The interday percentage of nominal value ranged from 85.61 to $114.69,85.61$ to 111.72, and 84.18 to 114.78 for PG, KPG, and HPG, respectively. Results are presented in Tables 3 for PG, KPG, and HPG, respectively.
Intraday accuracy and precision

Analyze replicate concentrations of PG in human $\mathrm{K}_{2}$ EDTA plasma performed intraday accuracy and precision evaluations. The run consisted of a CC plus a total of 24 spiked samples; six replicates each of LLOQ, LQC, MQC, and HQC samples.

The intraday CV ranged from 1.60 to $7.88,1.96$ to 8.82 , and 1.17 to 7.05 for PG, KPG, and HPG, respectively. The intraday percentage of nominal value ranged from 92.81 to $114.69,87.82$ to 111.72 , and 93.66 to 114.26 for PG, KPG, and HPG. Results are presented in Table 4, respectively.

\section{Dilution integrity}

Dilution QC is diluted 5th and 10th in human K EDTA plasma. Before extraction, six samples each of $5^{\text {th }}$ and $10^{\text {th }}$ diluted samples were processed and analyzed with freshly processed calibration samples. The calculated concentrations, including the dilution factor for $1 / 5^{\text {th }}$ and 
Table 1: Mass spectrometric conditions

\begin{tabular}{ll}
\hline Name & Condition \\
\hline Curtain gas (CUR) & $25 \mathrm{psi}$ \\
Collision gas (CAD) & Medium \\
Ion spray voltage (IS) & $5500 \mathrm{~V}$ \\
Turbo probe temperature & $400^{\circ} \mathrm{C}$ \\
Nebulizer gas (Gas 1) & $35 \mathrm{psi}$ \\
Heater gas (Gas 2) & $40 \mathrm{psi}$ \\
EP & $10 \mathrm{~V}$ \\
Dwell time & $200 \mathrm{~ms}$ \\
Resolution & Q1: Unit \\
& Q3: Unit \\
\hline
\end{tabular}

EP: Entrance potential

Table 2: Summary of the chromatographic conditions

\begin{tabular}{ll}
\hline Name & Values \\
\hline Analytical column & Xterra RP18 100×4.6 mm \\
Mobile phase & $10 \mathrm{mM}$ ammonium formate: \\
& methanol [1]:acetonitrile[1] - 30:70, v/v \\
Injection volume & $20 \mu \mathrm{L}$ \\
Flow rate & $0.7 \mathrm{~mL} / \mathrm{min}$ \\
Run time & $4.00 \mathrm{~min}$ \\
$\begin{array}{l}\text { Column oven } \\
\text { temperature }\end{array}$ & $40^{\circ} \mathrm{C}$ \\
Auto sampler & \\
temperature & $10^{\circ} \mathrm{C}$ \\
Retention times (min) & \\
Analyte & Pioglitazone \\
& Keto pioglitazone \\
& Hydroxy 2.10 \\
& pioglitazone \\
Internal standard & Diphenhydramine \\
\hline
\end{tabular}

1/10th yielded CV for PG, KPG, and HPG are 1.58-3.79, 2.16-3.17, and 3.07-3.74, respectively. Percentages of nominal values for $1 / 5$ th and 1/10th dilution for PG, KPG, and HPG are 96.71-112.37, 89.04-90.93, and 97.43-101.76, respectively.

\section{Ruggedness}

Ruggedness was performed by the different analyst using new or different column. The run consisted of a CC and a total of 24 spiked samples; six replicate each of the LLOQ LQC, MQC, and HQC samples. The within-run \% coefficients of variation ranged from 1.95 to 3.86 , 2.32 -to 6.78 , and 2.39 -to 3.01 for PG, KPG, and HPG, respectively. The percentage of nominal values ranged from 92.59 to $111.38,85.61$-to 104.85, and 92.61 -to 113.01 for PG, KPG, and HPG, respectively.

\section{Recovery}

Recovery of PG, KPG, and HPG was evaluated by comparing mean analyte responses of six extracted samples of LQC, MQC, and HQC samples to mean analyte response of six replicates injection of unextracted QC samples.

For PG mean recovery values are $83.95 \%, 82.35 \%$, and $80.98 \%$ at the HQC, MQC, and LQC samples, respectively. For KPG mean recovery values are $83.50 \%, 82.84 \%$, and $79.52 \%$ at the HQC, MQC, and LQC samples, respectively. For HPG mean recovery values are $70.03 \%$, $73.82 \%$, and $69.89 \%$ at the HQC, MQC, and LQC samples, respectively. Results are presented in Tables 5 for HQC, MQC, and LQC levels of PG, $\mathrm{KPG}$, and $\mathrm{HPG}$, respectively.

For the IS mean IS responses of 18 extracted samples were compared to the mean IS responses of 18 injections (6 replicates injections from each LQC, MQC, and HQC) of unextracted IS samples.

Mean recovery value for the IS diphenhydramine is $78.63 \%$. Results are presented in Tables 6 .

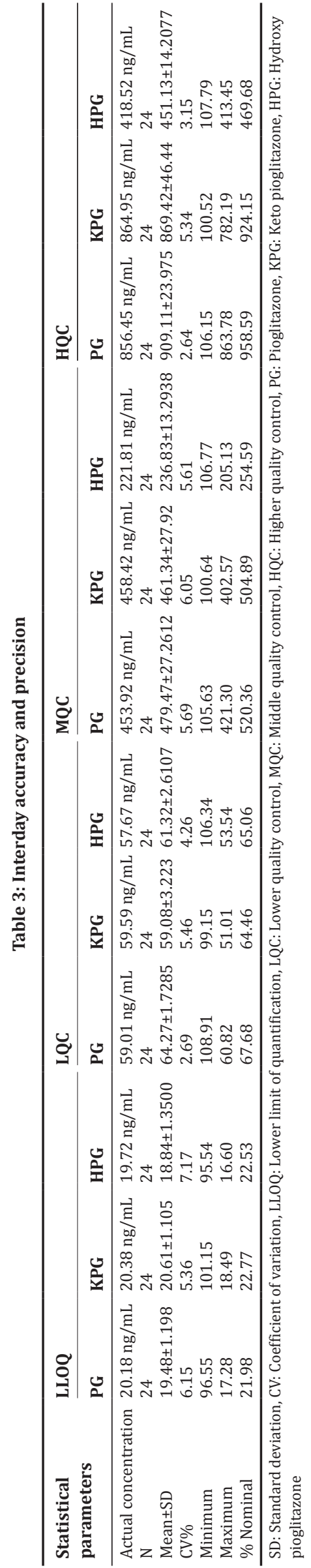



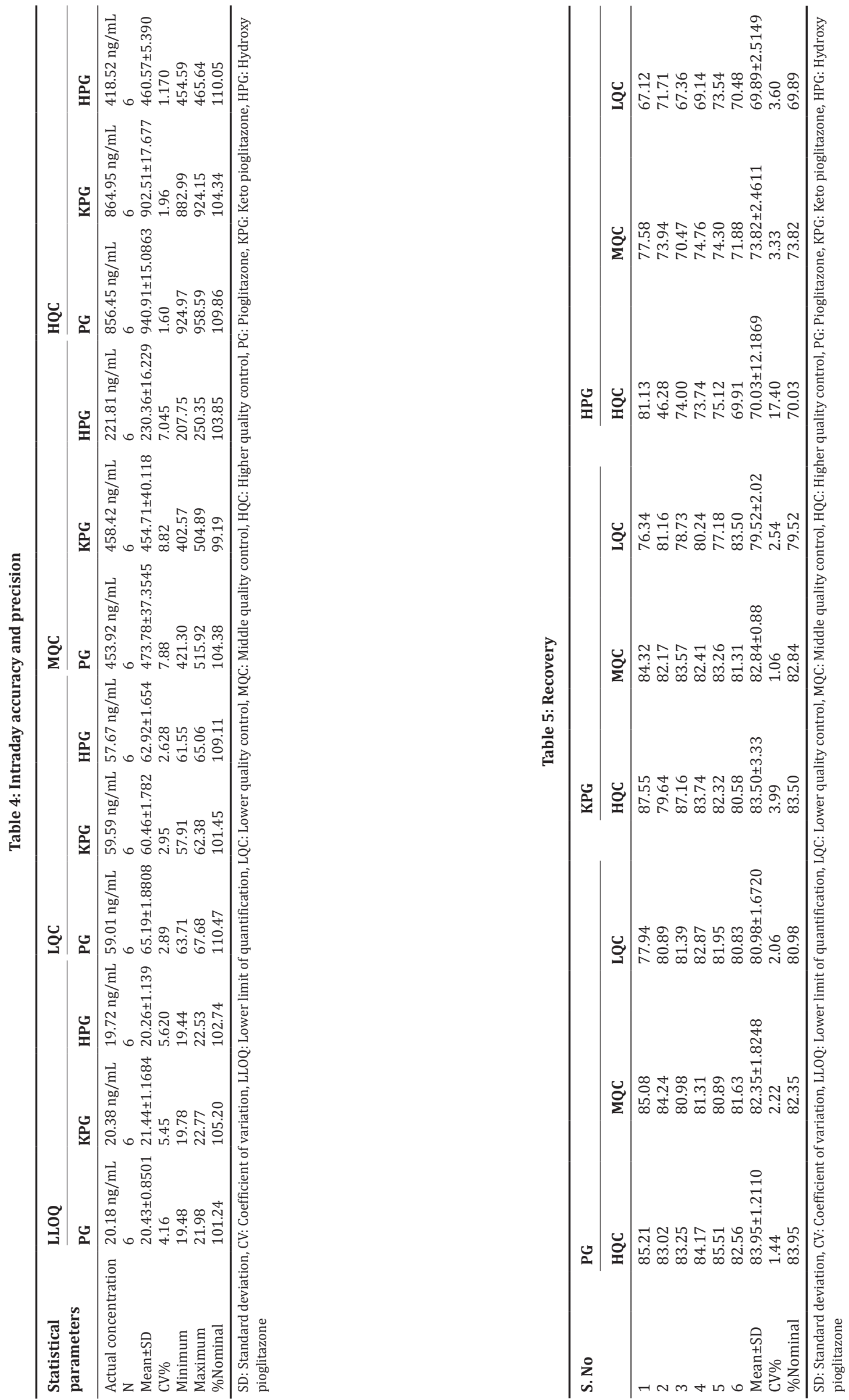
Table 6: Recovery of IS

\begin{tabular}{|c|c|c|c|}
\hline S. No & AQ IS area & Extracted IS area & \% Recovery \\
\hline 1 & 436166 & 351089 & 80.49 \\
\hline 2 & 441244 & 349938 & 79.31 \\
\hline 3 & 449645 & 351481 & 78.17 \\
\hline 4 & 451643 & 344104 & 76.19 \\
\hline 5 & 442404 & 350604 & 79.25 \\
\hline 6 & 447495 & 337328 & 75.38 \\
\hline 7 & 417311 & 329016 & 78.84 \\
\hline 9 & 439305 & 346389 & 78.85 \\
\hline 10 & 445262 & 349456 & 78.48 \\
\hline 11 & 448832 & 341730 & 76.14 \\
\hline 12 & 446844 & 358121 & 80.14 \\
\hline 13 & 439493 & 358613 & 81.60 \\
\hline 14 & 443280 & 343225 & 77.43 \\
\hline 15 & 443942 & 353001 & 79.52 \\
\hline 16 & 438181 & 343411 & 78.37 \\
\hline 18 & 441984 & 353801 & 80.05 \\
\hline Mean \pm SD & $442580.56 \pm 7835.4874$ & $347955.39 \pm 8145.9950$ & $78.63 \pm 1.6559$ \\
\hline $\mathrm{CV} \%$ & 1.77 & 2.34 & 2.11 \\
\hline Mean recovery & & & 78.63 \\
\hline
\end{tabular}

SD: Standard deviation, CV: Coefficient of variation, IS: Internal standard

\section{Stability}

\section{Short-term stock solution stability}

Two portions of solutions for analytes (PG, KPG, and HPG) and ISs (diphenhydramine) from the stock solutions taken. The solutions kept on the bench as such at room temperature and in the deep freezer for approximately $7.00 \mathrm{hrs}$. Fresh stock solution (comparison samples) for analytes (PG, KPG, and HPG) and ISs (diphenhydramine) prepared. Then, six vials (each from bench top, deep freezer, and freshly prepared for both drug and ISs) at SSMQC level prepared. Give six replicates injection from each vial and use area response to determine \% change over time.

PG, KPG, and HPG, and ISs (diphenhydramine) are found to be stable in reconstitution solution, on the bench at room temperature and in the deep freezer for approximately $6.00 \mathrm{hrs}$.

For PG, KPG, and HPG the \% change found are $-3.26,-0.63$, and 1.77 on bench top $-1.72,-1.89$, and 1.45 in deep freezer, respectively. For ISs diphenhydramine, the $\%$ change found are 0.16 on bench top -1.04 in deep freezer, respectively.

\section{Bench top stability}

Six samples each of LQC and HQC (stability samples) was kept on the bench at room temperature for approximately $6.30 \mathrm{hrs}$. Stability samples were processed and analyzed along with freshly processed calibration and comparison samples (six samples each of LQC and HQC). Concentrations were calculated to determine \% change over time.

PG, KPG, and HPG are found to be stable for $6.30 \mathrm{hrs}$ at room temperature. The \% change of PG, KPG, and HPG for LQC is $-2.58,-1.86$, and -3.67 , respectively, and for $\mathrm{HQC}$ is $2.95,6.25$, and 1.06 , respectively.

\section{Freeze-thaw stability}

Six samples each of low and high QC were retrieved from $-70 \pm 5^{\circ} \mathrm{C}$ after $24 \mathrm{hrs}$ of storage of samples. After thawing the stability, samples were restored for at least $12 \mathrm{hrs}$, and again the same samples were retrieved and kept on the bench at room temperature for thaw. The samples were restored, and after at least $12 \mathrm{hrs}$ again retrieve and thawed. Six stability samples (after three cycles) and six comparison samples at each level (LQC and HQC) were processed and analyzed along with freshly processed calibration samples. Concentrations were calculated to determine $\%$ change over time.
PG, KPG, and HPG are found to be stable for three freeze-thaw cycles at $-70 \pm 5^{\circ} \mathrm{C}$. The $\%$ change of PG, KPG, and HPG for LQC is $6.84,-0.66$, and -0.31 , respectively, and for HQC is $0.14,-3.59$, and -4.02 , respectively.

\section{Autosampler stability}

Six samples (stability samples) each of LQC and HQC were processed and kept in an auto sampler at $10^{\circ} \mathrm{C}$ for approximately $35.00 \mathrm{hrs}$. The stability samples were analyzed along with freshly processed calibration and comparison samples (six samples each of LQC and HQC). Concentrations were calculated to determine \% change over time. PG, $\mathrm{KPG}$, and HPG are found to be stable for $35.00 \mathrm{hrs}$ at auto sampler $10^{\circ} \mathrm{C}$. The \% change of PG, KPG, and HPG for LQC is $-1.80,4.58$, and 2.71, respectively, and for $\mathrm{HQC}$ is $-2.59,-1.62$, and -0.85 , respectively.

\section{Dry extract stability}

Six samples each of LQC and HQC samples were processed up to dry extract stage and stored in a deep freezer maintained at $-70 \pm 5^{\circ} \mathrm{C}$ for approximately $24.00 \mathrm{hrs}$ (stability samples). Stability samples were reconstituted and analyzed along with freshly processed CC and six replicates of LQC and HQC samples (comparison samples). Concentrations were calculated to determine $\%$ change over time.

PG, KPG, and HPG are found to be stable for $24.00 \mathrm{hrs}$ at room temperature.

The \% change of PG, KPG, and HPG for LQC is $-0.58,8.04$, and -0.91 , respectively, and for HQC is 7.12, 8.60, and 3.96, respectively.

\section{Reconstitution solution stability}

Six samples (stability samples) each of LQC and HQC level and keep it on bench top at room temperature for at least six hrs. The stability samples analyzed along with freshly processed calibration, LQC and HQC control samples (comparison samples). Calculate concentrations to determine \% change over time. PG, KPG, and HPG are found to be stable for approximately $6.00 \mathrm{hrs}$ at room temperature.

The \% change of PG, KPG, and HPG for LQC is $-9.83,-8.05$, and -3.67 , respectively, and for HQC is $-8.63,-9.33$, and -6.09 , respectively.

\section{Matrix effect}

Eighteen blank matrix samples from six different lots of matrix are processed. The reconstituted blank samples spiked with HQC and LQC level (from each lot one blank, one HQC and one LQC sample) 
and compared against corresponding aqueous $\mathrm{HQC}$ and $\mathrm{LQC}$ sample injected in six replicates. No effect of quantitation for PG, KPG, and HPG, and IS (diphenhydramine) was observed.

\section{Reinjection reproducibility}

After the analysis of any precision and accuracy batch, re inject the QC samples from the last three sets (LQC and HQC) and calculate the concentration. The back calculated concentrations of the HQC and LQC samples using the CC of the same precision and accuracy batch. The \% change of PG, KPG, and HPG for LQC is 2.96, 2.11, and 2.03, respectively, and for $\mathrm{HQC}$ is $-0.79,5.02$, and -0.55 , respectively.

\section{CONCLUSION}

The particular LC-MS/MS assay shown with this report is simple, fast and sensitive pertaining to the determination of PG, KPG, and HPG in human plasma. This method is fully validated as per the recent FDA guidelines. This process makes use of Diphenhydramine as IS intended for quantification avoiding possible matrix influence associated difficulties in addition to variability in extraction efficiency between the analyte and the IS. The straightforward LLE technique is consistent and also reproducible recoveries for the analyte along with the IS from plasma. Furthermore, the whole analysis time period (extraction in addition to chromatography) would be the smallest compared to most of these reported procedures. The method furnished beneficial linearity and being trusted to guide pharmacokinetic study in humans. In the outcomes of all the agreement parameters, the proposed assay can be useful regarding bioavailability in addition to bioequivalence studies and therapeutic drug monitoring while using the ideal perfection in addition to reliability.

\section{ACKNOWLEDGMENTS}

The authors gratefully acknowledge Src Laboratories Private Limited (Hyderabad, India), for providing essential services to carry out this specific operate.

\section{REFERENCES}

1. Al-Majed A, Bakheit AH, Abdel Aziz HA, Alharbi $\mathrm{H}$, AlJenoobi FI. Pioglitazone. Profiles Drug Subst Excip Relat Methodol 2016;41:379-438.

2. El-Ghobashy M, Yehia A, Mostafa A. Application of membraneselective electrodes for the determination of pioglitazone $\mathrm{HCl}$ in the presence of its acid degradant or metformin $\mathrm{HCl}$ in tablets and plasma. Anal Lett 2009;42:123-40.

3. Faridbod F, Ganjali MR, Nasli-Esfahani E, Larijani B, Riahi S, Norouzi P, et al. Potentiometric sensor for quantitative analysis of pioglitazone hydrochloride in tablets based on theoretical studies. Int J Electrochem Sci 2010;5:880-94.

4. Ali MY, Swamy PV, Borgaonkar P, Raju SA. UV-spectrophotometric determination of pioglitazone in pharmaceutical dosage forms. Int $\mathrm{J}$ Chem Sci 2008;6:2062-5.

5. Saber AM. Determination of pioglitazone hydrochloride in tablets by high-performance liquid chromatography. Pak J Anal Environ Chem 2008;9:118-21.

6. Radhakrishna T, Sreenivas Rao D, Om Reddy G. Determination of pioglitazone hydrochloride in bulk and pharmaceutical formulations by HPLC and MEKC methods. J Pharm Biomed Anal 2002;29(4):593-607.

7. Abdelmonem AA, Ragab GH, Hashem HA, Bahgat EA. High performance liquid chromatographic and spectrophotometric determinations of pioglitazone- $\mathrm{HCl}$ either alone or in combination with metformine-HCl. J Liq Chromatogr Relat Technol 2012;35:2706-23.

8. Zhong WZ, Lakings DB. Determination of pioglitazone in dog serum using solid-phase extraction and high-performance liquid chromatography with ultraviolet $(229 \mathrm{~nm})$ detection. J Chromatogr 1989;490(2):377-85

9. Yamashita K, Murakami H, Okuda T, Motohashi M. High-performance liquid chromatographic determination of pioglitazone and its metabolites in human serum and urine. J Chromatogr B Biomed Appl 1996;677(1):141-6.

10. Zhong WZ, Williams MG. Simultaneous quantitation of pioglitazone and its metabolites in human serum by liquid chromatography and solid phase extraction. J Pharm Biomed Anal 1996;14(4):465-73.

11. Lakshmi NY, Barhate VD. Development and validation of stability indicating UPLC method for the simultaneous determination of anti-diabetic drugs in pharmaceutical dosage forms. J Pharm Res 2010;3(12):3081-7.

12. Karra VK, Pilli NR, Inamadugu JK, Rao JS. Simultaneous determination of pioglitazone and candesartan in human plasma by LC-MS/MS and its application to a human pharmacokinetic study. J Pharm Anal 2012;2(3):167-73

13. Lin ZJ, Ji W, Desai-Krieger D, Shum L. Simultaneous determination of pioglitazone and its two active metabolites in human plasma by LCMS/MS. J Pharm Biomed Anal 2003;33(1):101-8.

14. Sripalakit P, Neamhom P, Saraphanchotiwitthaya A. High-performance liquid chromatographic method for the determination of pioglitazone in human plasma using ultraviolet detection and its application to a pharmacokinetic study. J Chromatogr B Analyt Technol Biomed Life Sci 2006;843(2):164-9

15. Tahmasebi E, Yamini Y, Saleh A. Extraction of trace amounts of pioglitazone as an anti-diabetic drug with hollow fiber liquid phase microextraction and determination by high-performance liquid chromatography-ultraviolet detection in biological fluids. J Chromatogr B Analyt Technol Biomed Life Sci 2009;877(20-21):1923-9.

16. Chinnalalaiah R, Pigili R, Avanapu SR. Liquid chromatography and tandem mass spectrometry method for quantitative determination of pioglitazone and its metabolite 5-hydroxy pioglitazone in human plasma. Ann Pharm Fr 2017;75(2):105-11.

17. Jagadeesh B, Bharathi DV, Pankaj C, Narayana VS, Venkateswarulu V. Development and validation of highly selective and robust method for simultaneous estimation of pioglitazone, hydroxypioglitazone and metformin in human plasma by LC-MS/MS: Application to a pharmacokinetic study. J Chromatogr B Analyt Technol Biomed Life Sci 2013;930:136-45.

18. Lakshmi PR, Prahlad P, Mastanamma SK, Ravindra N, Rao MV. UPLC separation analysis of emtricitabine, tenofovir, cobicistat and elvitegravir from their degradation products. Int J Pharm Pharm Sci 2016;8(4):362-9

19. Lakshmi PR, Prahlad P, Mastanamma SK, Ravindra N, Rao MV. Development and validation of a stability indicating reverse phase high performance liquid chromatography method for simultaneous determination of clindamycin, metronidazole, and clotrimazole in pharmaceutical combined dosage forms. Asian J Pharm Clin Res 2017;10(1):1-7.

20. US DHHS, FDA, CDER. Guidance for Industry: Bioanalytical Method Validation. Rockville, MD: US Department of Health and Human Services, Food and Drug Administration, Center for Drug Evaluation and Research and Center for Veterinary Medicine; 2001.

21. European Medicines Agency. Guideline on Bioanalytical Method Validation, Science and Medicinal Health. European Medicines Agency (EMEA), EMEA, CHMP, EWP/192217; 2009. 